\title{
A Review of the Robotic Radical Prostatectomy Outcomes
}

\section{Suhani Maharajh}

\begin{abstract}
Robotic radical prostatectomy was first performed in 2000 . The superior range of movement as well as better ergonomics were clear advantages of this technology. This technology and its cost can however only be justified, if it has clear data confirming its equivalence or preferably superiority in management. Open radical retropubic prostatectomy has previously been the gold standard for oncological outcomes in organ confined prostate cancer. Follow-up data showed good 5-year cancer free survival and mortality and Walsh's technique contributed significantly to improvement of quality of life issues, such as sexual function. In spite of improved survival rates with other less invasive modalities, such as brachytherapy and intensity-modulated radiotherapy, the radical prostatectomy is still a popular treatment choice as there is long-term data to support evidence of durable cancer control. The recovery and cost of the open procedure has often been a disadvantage. Minimal access surgery was able to overcome these issues with good surgical outcomes. Robotic surgery may well be the next step in surgical and technological revolution and has the possibility of making minimally invasive surgery accessible to surgeons with less laparoscopic experience. This review will attempt to assess the outcomes of robotic surgery to determine if it is indeed a feasible option for the treatment of organ confined prostate cancer, by assessing the surgical outcomes.
\end{abstract}

Keywords: Robotic radical prostatectomy, Outcomes, Trifecta, Continence, Erectile function, Complications, Oncologic outcomes, Positive surgical margins.

How to cite this article: Maharajh S. A Review of the Robotic Radical Prostatectomy Outcomes. World J Laparosc Surg 2013;6(2):69-73.

\section{Source of support: Nil}

Conflict of interest: None declared

\section{INTRODUCTION}

With the evolution of disease patterns, increased awareness around screening and widespread availability of prostatespecific antigen (PSA), prostate cancer is increasingly being recognized at the stage of organ confined disease. ${ }^{1}$ It is a disease associated with the elderly males, who often have comorbid conditions. ${ }^{2}$ Screening from the age of 40 , as per the EUA recommendations, has resulted in younger males in the sexually active age group being diagnosed with this disease. Preservation of continence and sexual function are both prime considerations in this age group of individuals. The previous gold standard of open radical prostatectomy has shown durable excellent oncologic outcomes, but due to the complexity of this procedure, quality of life issues such as erectile function and continence rates, have produced variable results, affected by the surgeon experience, amount of annual surgeries in the respective centers as well as patient and cancer-specific variables.

The laparoscopic procedure with better visualization of nerve anatomy and superior reconstruction of the pelvic floor anatomy has shown good results over the past years. ${ }^{3}$ The procedure has a considerable learning curve in spite of its clear benefits of lower hospital stay and transfusion rate. ${ }^{3}$ However, technology may offer an advantage in terms of the learning curve. The introduction of robotic surgery by Da Vinci robotic surgery (Fig. 1) has made these operative skills more attainable goal with a reportedly less steep learning curve. Despite the lower hospital stay with robotassisted radical prostatectomy (RARP), the considerable cost still overshadows any apparent benefit to the technology. ${ }^{3}$

However, if evidence-based evaluation were able to show clear benefits in terms of oncological and quality of life outcomes, the cost could be at least justified, in the limited centers that are able to afford this revolutionary technology.

This article will review the recent literature regarding robotic radical prostatectomy, and examine whether it is a superior treatment option in the treatment of organ confined prostate cancer.

\section{MATERIALS AND METHODS}

Keywords pertaining to the topic were used to identify relevant literature, through the use of search engines (PubMed Central, Google scholar and Medline). The MESH keywords utilized included the following:

- Robotic radical prostatectomy (Fig. 2)

- Outcomes

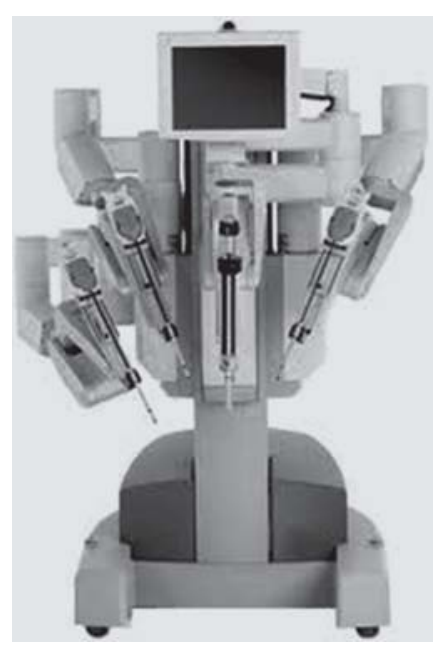

Fig. 1: da Vinci robot (Source: http://www.daviscrump. com/) 


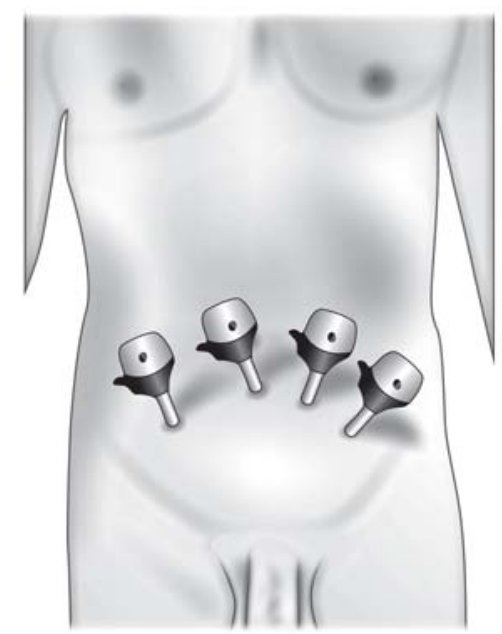

Fig. 2: Port placement in robotic radical prostatectomy (Source: http://www.avantgardeurology.com/)

- Trifecta

- Continence

- Erectile function

- Complications

- Oncologic outcomes

- Positive surgical margins.

Relevant articles identified through the previously mentioned search engines were identified. MESH keywords were to identify the most relevant articles, and only these articles are included in the review. Fifteen articles were included in the review. Prospective and retrospective studies were both included in this review. In addition, relevant articles were cross-referenced from the articles identified. Cohort studies were also used, which compared open and laparoscopic approaches to the robotic radical prostatectomy outcome. Only English articles that were published from 2010 until current date were included for analysis.

Results were analyzed by the author and summarized in data capture sheet for analysis.

Endnote citation manager was used for the purposes of referencing and downloaded directly from the respective articles and search engines.

\section{RESULTS}

Eight articles were identified that dealt with complications. Not all articles defined whether complications were minor or major, whether transfusions were included and whether or not they were graded according to the Clavien system or not (Table 1).

The following 10 studies includes one study by Kaffenberger ${ }^{11}$ et al that assessed the outcomes in salvage prostatectomy postablative therapy (Table 2).

There were different definitions of failure and recurrence in each of the studies below. The duration of follow-up also varied as specified in each case (Table 3).
Erectile preservation rates differed based on age, preoperative erectile function, nerve sparing techniques and the early use of PDE-5 inhibitors, accounting for the differences seen below (Table 4).

Differences in preservation of the puboprostatic ligaments, the use of the Rocco suture and reconstruction of the posterior rhabdosphincter, account for the differences in rates below (Table 5).

\section{DISCUSSION}

Prostate carcinoma is now the most common solid neoplasm of the male population. ${ }^{18}$ With a shift of the population dynamics in the developed, world as well as the widespread awareness of PSA ${ }^{2}$ and prostate cancer and Men's health awareness, there has been a shift in the profile of diagnosed patients. Prostate carcinoma is a major cause of morbidity and mortality and is now increasingly being diagnosed in male patients at an earlier stage when it is organ-confined. ${ }^{2}$

Disease has physical, social and psychological ramifications on the well-being of an individual. This is extremely relevant in the treatment of prostate cancer as it has the potential to have debilitating consequences on all of these aspects of health, as the outcomes of surgery can have adverse effects on sexual and voiding function, with adverse psychological sequelae and patient dissatisfaction. ${ }^{19}$

The trifecta outcome ${ }^{20}$ is used to describe the triad of oncological, potency and continence outcomes. These areas are important to assess outcomes in surgery. Quality of life

\begin{tabular}{lc}
\multicolumn{2}{c}{ Table 1: Complication rate results } \\
\hline References & Complications (\%) \\
\hline Bouchier-Hayes $^{4}$ et al $(n=125)$ & 12.8 \\
Doumerc $^{5}$ et al $(n=212)$ & 1.8 \\
Jayram $^{6}$ et al $(n=148)$ & 3.4 \\
Padanavo $^{7}$ et al $(n=153)$ & 7.8 \\
Patel $^{8}$ et al $(n=1,100)$ & 8.6 \\
Sharma $^{9}$ et al $(n=500)$ & 5.6 \\
Yip $^{10}$ et al $(n=235)$ & 7 \\
\hline
\end{tabular}

$\mathrm{n}$ : patient numbers in individual studies

\begin{tabular}{lc}
\multicolumn{2}{c}{ Table 2: Results for positive surgical margins } \\
\hline References & $\begin{array}{c}\text { Positive surgical } \\
\text { margins }(\%)\end{array}$ \\
\hline Bouchier-Hayes $^{4}$ et al $(n=125)$ & 32 \\
Doumerc $^{5}$ et al $(n=212)$ & 21.3 \\
Jayram $^{6}$ et al $(n=148)$ & 20.9 \\
Kaffenberger $^{11}$ et al $(n=34)$ & 26 \\
Leitao $^{12}$ et al $(n=171)$ & 23.4 \\
Novara $^{13}$ et al $(n=242)$ & 31 \\
Padanavo $^{7}(n=153)$ & 35 \\
Patel $^{8}$ et al $(n=1,100)$ & 10.6 \\
Sharma & 9 et al $(n=500)$ \\
Yip $^{14}$ et al $(n=235)$ & $23-26$ \\
\hline
\end{tabular}

$\mathrm{n}$ : patient numbers in individual studies 
Table 3: Summary of incidence of biochemical recurrence and failure

\begin{tabular}{lll}
\hline References & Biochemical recurrence (\%) & Biochemical failure (\%) \\
\hline Bouchier-Hayes $(n=125)$ & $7.6(12$ months $)$ & 18 (16 months) \\
Kaffenberger $^{15}(n=34)$ & $15.7(60$ months $)$ & \\
Liss $^{16}(n=289)$ & $21.3(24$ months $)$ & 2 (9 months) \\
Jayram $^{6}(n=148)$ & & \\
Padanavo $^{7}(n=153)$ &
\end{tabular}

$\mathrm{n}$ : patient numbers in individual studies

issues can no longer be viewed as separate entities from disease treatment and side effects. Radical retropubic prostatectomy has been previously performed by the open surgical route and after the detailed anatomic description of nerve sparring radical prostatectomy by Walsh, has had good outcomes in this regard. ${ }^{8}$ However, as result of improvements in minimal access surgery, similar outcomes have been reported in the literature with laparoscopic radical prostatectomy. ${ }^{21}$

The first robotic-assisted laparoscopic surgery was performed in 2001. ${ }^{21}$ Data has confirmed that there is a shorter recovery, shorter hospital stay and a lower transfusion rate ${ }^{13}$ with the procedure, but this must be counterbalanced against the high operating and maintenance costs of the robot. ${ }^{3}$

The complication rates in this review varied from 1.8 to $12.8 \%$. This is comparable to the accepted complication rate in open retropubic radical prostatectomy, the previously considered gold standard. The articles did not include a more

\begin{tabular}{lc}
\multicolumn{2}{c}{ Table 4: Preservation of erectile function } \\
\hline References & $\begin{array}{c}\text { Erectile function } \\
\text { preservation }(\%)\end{array}$ \\
\hline Bouchier-Hayes $^{4}$ et al $(n=125)$ & 72 \\
Decastro $^{17}$ et al $(n=140)$ & 60 \\
Jayram $^{6}$ et al $(n=148)$ & 51.7 \\
Leitao $^{12}$ et al $(n=171)$ & 69.1 \\
Novara $^{13}$ et al $(n=242)$ & 60 \\
Patel $^{14}$ et al $(n=1,100)$ & 96.6 \\
Sharma & et al $(n=500)$ \\
Yip $^{10}$ et al $(n=235)$ & 75 \\
\hline
\end{tabular}

$\mathrm{n}$ : patient numbers in individual studies

\begin{tabular}{lc}
\multicolumn{2}{c}{ Table 5: Preservation of continence } \\
\hline References & $\begin{array}{l}\text { Continence } \\
\text { preservation (\%) }\end{array}$ \\
\hline Bouchier-Hayes $^{4}(n=125)$ & 93 \\
Decastro $^{17}(n=140)$ & 55.7 \\
Jayram $^{6,11}(n=148)$ & 91.5 \\
Kaffenberger $^{11}(n=34)$ & 39 \\
Leitao $^{12}(n=171)$ & 90.3 \\
Novara $^{13}(n=242)$ & 89 \\
Padanavo $^{7}(n=153)$ & 98 \\
Patel $^{8}(n=1,100)$ & 97.9 \\
Sharma & $(n=500)$ \\
Yip $^{10}(n=235)$ & 91.3 \\
\hline
\end{tabular}

$\mathrm{n}$ : Patient numbers in individual studies detailed breakdown in these complications as to ascertain the degree of morbidity. Three of the studies were from centers that involved the first 200 cases, and hence incorporate the learning curve and are therefore still encouraging, that there was no statistically significant increase in the incidence of the complications, even with less experienced surgeons. There were also no reported mortalities in these studies.

Positive surgical margins ranged from 10.6 to $35 \%$. The reported prevalence in the literature ranges from 10 to $45 \%{ }^{9}$ This is therefore in the acceptable range. Of note, is that in the study by Kaffenburger et al ${ }^{15}$ which assessed positive surgical margins in salvage radical prostatectomy, positive surgical margin rate was $26 \%$. This suggests that even in patients that failed previous modalities, RARP still has an acceptable positive surgical margin ${ }^{21}$ rate that is comparable to that of cases where radical prostatectomy was offered at the outset of treatment. However, the fact that this has a smaller representative patient population makes this data difficult to interpret. The Gleason grade and D'Amico classification was not specified in all series, although there was correlation noted by specific groups between higher stage disease, positive surgical margins and high risk disease. This has been confirmed by review of the current literature as well. ${ }^{22}$

Although positive surgical margins were noted, this did not correlate with the disease recurrence. Pandanavo ${ }^{7}$ et al had two recurrences, both of whom had negative surgical margins. Across these studies the apex and base were the most common sites of recurrence. This concurs with previous literature. It also correlates with the PSA and the prostate volume as well. ${ }^{10}$ It would be of value to know if these cases of positive surgical margins were confined to cases of nerve sparing techniques, but this information was not specified.

With regards to biochemical recurrence and failure, it is difficult to standardise and analyze this data, as different follow-up periods were used. The incidence however did correlate with higher stage disease ${ }^{23}$ and has similar rates to other studies. ${ }^{24}$

The erectile preservation range varied across the studies. Of note, the high volume center with $1,100^{8}$ cases had excellent potency rates, in spite of using a strict definition 
$\mathrm{n}$ (erections adequate for intercourse with use of phosphodiesterase- 5 inhibitors, but not other assistive devices). This would suggest that more experience would improve potency outcomes. However, this could also be due to the patient profile undergoing robotic retropubic radical prostatectomy in this series. Also the study did note a better potency rate in younger patients, and this could therefore have a significant impact on outcomes. The range reported here is consistent with that in literature of studies with nerve preserving radical prostatectomies. ${ }^{8}$ However, most of these patients had concomitantly used phosphodiesterase-5 inhibitors. The use of early rehabilitation of erectile function was not specified across all these studies, and it is therefore difficult to compare these results. More literature, and a randomized controlled trial on the use of phosphodiesterase- 5 inhibitor's impact on outcomes of erections in bilateral vs unilateral nerve sparing procedures, would be helpful in this regard. Most of these techniques used athermal dissection and the principles of nerve sparing procedures (although not specified). In addition the use of the intrafascial $v s$ interfascial dissection planes of nerve dissection affects potency outcome. The specifics of this were not provided and hence its impact on outcomes cannot, be assessed in this review. However, different tools were used as assessment of the erectile function. Most often questionnaires were used. The outcomes are therefore, reporter dependant, and therefore reliability of this is, patient dependant.

The continence rate in this review is consistent with the rates reported in the literature. ${ }^{8}$ The one study by Kaffenberger et $\mathrm{al}^{11}$ reports a considerably lower rate of continence of 39\%. However, it must be borne in mind that this was in a series of salvage radical prostatectomies, postablative treatment. This may impact on the surgical outcomes. Across the studies, the definition of continence differed with some defining it as pad free ${ }^{8}$ and the rest allowing for one pad. There were differences in the duration of follow-up, as well. Most studies were followed up until at least 1 year. Prolonged follow-up should not provide an improved rate of continence noted, however. There were also differences in the use of continence preservation techniques in the different series.

Patel et $\mathrm{al}^{8}$ reconstructed the rhabdosphincter and used periurethral suspension stitches. This may have contributed to the excellent continence rates noted in this large series. ${ }^{12}$ Leitao et $\mathrm{al}^{12}$ preserved the puboprostatic ligaments and these may contribute to their results.

\section{CONCLUSION}

RARP has outcomes that are similar to laparoscopic radical prostatectomy. The cost can however be a prohibitive factor.
Although the outcomes of this procedure have curtailed the inhospital stay, it cannot counterbalance the cost of this procedure until sufficient data shows superior outcomes of this procedure. This review shows that although there is no sacrifice of trifecta outcomes, no clear benefit over conventional laparoscopy was demonstrated. Studies that directly compare the learning curve of robotic radical prostatectomy and laparoscopic radical prostatectomy may help in settling this debate and providing motivation for robotic radical retropubic prostatectomy.

\section{REFERENCES}

1. Nordstrom T, Aly M, Clements MS, Weibull CE, Adolfsson J, Gronberg H. Prostate-specific antigen (PSA) testing is prevalent and increasing in Stockholm County, Sweden, despite no recommendations for PSA screening: Results from a populationbased study, 2003-2011. Eur Urol 2013;63(3):419-25.

2. Sapira MK, Obiorah CC. Age and pathology of prostate cancer in South-Southern Nigeria: Is there a pattern? Med J Malaysia 2012;67(4):417-19.

3. Bolenz C, Gupta A, Hotze T, Ho R, Cadeddu JA, Roehrborn CG, et al. Cost comparison of robotic, laparoscopic, and open radical prostatectomy for prostate cancer. Eur Urol 2010; 57(3):453.

4. Bouchier-Hayes DM, Clancy KX, Canavan K, O'Malley PJ. Initial consecutive 125 cases of robotic assisted laparoscopic radical prostatectomy performed in Ireland's first robotic radical prostatectomy centre. Ir J Med Sci 2012;181(1):21-25.

5. Doumerc N, Yuen C, Savdie R, Rahman MB, Rasiah KK, Pe Benito R, et al. Should experienced open rostatic surgeons convert to robotic surgery? The real learning curve for one surgeon over 3 years. BJU Int 2010;106(3):378-84.

6. Jayram G, Decastro GJ, Large MC, Razmaria A, Zagaja GP, Shalhav AL, et al. Robotic radical prostatectomy in patients with high-risk disease: A review of short-term outcomes from a high-volume center. Journal of Endourology 2011;25(3): 455-57.

7. Padavano J, Shaffer L, Fannin E, Burgers J, Poll W, Ward E, et al. Robotic radical prostatectomy at teaching community hospital: Outcomes and safety. JSLS 2011;15(2):193-99.

8. Patel VR, Coelho RF, Chauhan S, Orvieto MA, Palmer KJ, Rocco B, et al. Continence, potency and oncological outcomes after robotic-assisted radical prostatectomy: Early trifecta results of a high-volume surgeon. BJU Int 2010;106(5):696-702.

9. Sharma NL, Papadopoulos A, Lee D, McLoughlin J, Vowler SL, Baumert H, et al. First 500 cases of robotic-assisted laparoscopic radical prostatectomy from a single UK centre: Learning curves of two surgeons. BJU Int 2011;108(5):739-47.

10. Yip KH, Yee CH, Ng CF, Lam NY, Ho KL, Ma WK, et al. Robot-assisted radical prostatectomy in Hong Kong: A review of 235 cases. J Endourol 2012;26(3):258-63.

11. Kaffenberger SD, Keegan KA, Bansal NK, Morgan TM, Tang $\mathrm{DH}$, Barocas DA, et al. Salvage robotic-assisted laparoscopic radical prostatectomy: A single institution, 5-year experience. J Urol 2013;189(2):507-13.

12. Leitão TP, Papatsoris AG, Mandron E. Extraperitoneal laparoscopic radical prostatectomy: A prospective 2-year single-surgeon experience with 171 cases. Arab J Urol 2012;10: 347-532. 
13. Novara G, Ficarra V, Mocellin S, Ahlering TE, Carroll PR, Graefen M, et al. Systematic review and meta-analysis of studies reporting oncologic outcome after robot-assisted radical prostatectomy. Eur Urol 2012;62(3):382-404.

14. Patel V, Coelho R, Rocco B, Orvieto M, Sivaraman A, Palmer K, et al. Positive surgical margins after robotic assisted radical prostatectomy: A multi-institutional study. J Urol 2011;186(2): 511-16.

15. Briganti A, Bianchi M, Sun M, Suardi N, Gallina A, Abdollah $\mathrm{F}$, et al. Impact of the introduction of a robotic training programme on prostate cancer stage migration at a single tertiary referral centre. The trifecta outcome in 300 consecutive cases of robotic-assisted laparoscopic radical prostatectomy according to D'Amico risk criteria: Robot-assisted radical prostatectomy: Five year oncological and biochemical outcomes. Salvage robotic assisted laparoscopic radical prostatectomy: A single institution, 5-year experience. BJU Int 2012;39(1):107-13.

16. Liss MA, Lusch A, Morales B, Beheshti N, Skarecky D, Narula N, et al. Robot-assisted radical prostatectomy: 5-year oncological and biochemical outcomes. J Urol 2012;188(6): 2205-10.

17. DeCastro GJ, Jayram G, Razmaria A, Shalhav A, Zagaja GP. Functional outcomes in African-Americans after robot-assisted radical prostatectomy. J Endourol 2012;26(8):1013-19.

18. Tewari AK, Srivastava A, Sooriakumaran P, Grover S, Desir S, Dev H, et al. Pathological outcomes and strategies to achieve optimal cancer control during robotic radical prostatectomy in Asian-Indian men. Indian J Urol 2011;27(3):326-30.
19. Eton DT, Lepore SJ. Prostate cancer and health-related quality of life: A review of the literature. Psychooncology 2002;11(4): 307-26.

20. Bianco FJ Jr, Scardino PT, Eastham JA. Radical prostatectomy: Long-term cancer control and recovery of sexual and urinary function (trifecta). Urology 2005;66(5 Suppl):83-94.

21. Mottrie A, Gallina A, De Wil P, Thüer D, Novara G, Ficarra V. Balancing continence function and oncological outcomes during robot-assisted radical prostatectomy (RARP). BJU Int 2011; 108(6b):999-1006.

22. Barry MJ, Gallagher PM, Skinner JS, Fowler FJ Jr. Adverse effects of robotic-assisted laparoscopic versus open retropubic radical prostatectomy among a nationwide random sample of medicare-age men. J Clin Oncol 2012;30(5):513-18.

23. Padavano J, Shaffer L, Fannin E, Burgers J, Poll W, Ward ES, et al. Robotic radical prostatectomy at a teaching community hospital: Outcomes and safety. JSLS 2011;15(2):193-99.

24. Masterson TA, Cheng L, Boris RS, Koch MO. Open vs roboticassisted radical prostatectomy: A single surgeon and pathologist comparison of pathologic and oncologic outcomes. Urol Oncol 2012 Jan 3. [Epub ahead of print].

\section{ABOUT THE AUTHOR}

\section{Suhani Maharajh}

Specialist Urologist, Department of Urology, Greys Hospital Pietermaritzburg Urology Complex, Town Bush Road, Kwa-Zulu Natal 3200, South Africa, e-mail: suhanimaharajh@ymail.com 